\title{
A Construction Kit for Electronic Textiles
}

\author{
Leah Buechley \\ Craft Technology Group, University of Colorado at Boulder \\ Department of Computer Science \\ buechley@cs.colorado.edu
}

\begin{abstract}
Construction kits have long been popular as educational artifacts, supporting and encouraging creative explorations of engineering and design; but to date, such kits have had little connection with the new and expanding field of electronic textiles (e-textiles). We believe that creating an "e-textile construction kit" could provide a powerful new medium to engage a diverse range of students in electrical engineering and computer science. This paper, then, describes a construction kit designed to introduce novices to electronics, computing and design via e-textiles. We describe each component of the kit, provide examples of constructions that were built with the kit, and examine the durability of these constructions. We conclude with a discussion of the results of preliminary user testing and an exploration of our plans for continued work in this area.
\end{abstract}

\section{Introduction}

Electronic-textile research is closely related to wearable computing research, but in many ways its own distinct field. Wearable computing explores technologies that are portable and attached to or carried on the body, but e-textile research has a slightly different focus: investigating electronic and computational technology that is imbedded into textiles. E-textile researchers strive to build devices that are as soft, flexible and comfortable as traditional cloth artifacts [1-4]. E-textiles may be wearable, but the class also includes items such as wall hangings, quilts and other pervasive fabric-based artifacts.

Building blocks, Legos ${ }^{\circledR}$, sewing kits, and Erector Sets ${ }^{\circledR}$ allow children and other novices to design and build their own model buildings, clothing and mechanical constructions; kits like chemical modeling sets help professional scientists visualize threedimensional structures; some professional engineers make use of Legos ${ }^{\circledR}$ or other kits to prototype their ideas. In short, construction kits have a rich history as useful and engaging educational, visualization, and prototyping tools.

The power of construction kits stems from the fact that they provide simple modules, the construction kit pieces, which can be combined in a multitude of ways. Well-chosen modules conceal some of the complexities of "real-life" engineering and design, but are basic enough to provide users with the freedom to build a wide range of constructions $[5,6]$.

This paper introduces a construction kit for electronic textiles that provides a means for designers of a variety of ages and skill levels to create robust etextile prototypes. The kit also serves as a unique platform that students and others can use to explore the integration of electronics and computation with clothing and other textiles.

We believe that e-textiles provide a powerful construction kit medium for a number of reasons. First, we hope that an e-textile kit may appeal to a different class of users than is normally drawn to computing or electronics. In particular, we are intrigued by the possibility that the kit may provide a means to attract more women and girls to these traditionally male dominated fields. Furthermore, the field of e-textiles is a growing and tremendously exciting engineering field. We hope to help inspire and educate the next generation of scientists and engineers that will be feeding this discipline. We also hope that our kit will prove to be a useful prototyping tool for today's engineers.

Our construction kit design allows users to realize complete e-textile garments, but we should mention that our contributions are primarily in the area of etextile hardware rather than software. We have developed novel means of packaging off-the-shelf electronic components so that they can be easily attached to cloth and to one another. Our kit facilitates computation, but our work in designing and implementing software control systems for it is still in the preliminary stages. 

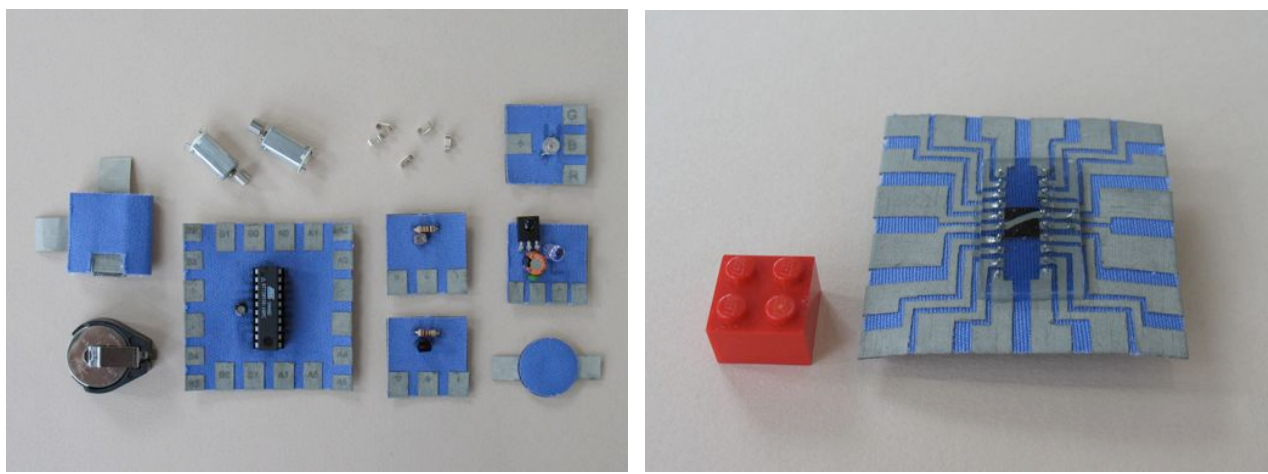

Figure 1. Left: the kit (needle, conductive thread and fabric paint not shown). Right: the underside of the stitch-able microcontroller.

\section{The Kit}

Figure 1 shows an image of our e-textile construction kit. This kit contains a microcontroller, an assortment of sensors and actuators, an infrared (IR) transceiver, an on/off switch and a battery pack. Each of these components is either made entirely of fabric or has been packaged so that it can be stitched to cloth. Components can be connected to one another electrically via traces sewn in conductive thread. Once a design is complete, exposed electrical traces can be insulated with fabric paint. The remainder of this section will examine each component of our kit in detail.

\subsection{Control: The Stitch-able Microcontroller and the Software Environment}

The central component of any e-textile design is its "brain", usually an embedded microcontroller. This section will introduce our e-textile microcontroller package, and will then briefly describe the software and hardware environments that we use to program the controllers.

Our stitch-able microcontroller package consists of a fabric printed circuit board (PCB), an integrated circuit (IC) socket, a filtering capacitor and a microcontroller. A top view of the stitch-able microcontroller can be seen in the left hand image in Figure 1. The right hand image in the figure displays its underside, showing the layout of the fabric PCB.

Our stitch-able microcontroller package holds a 20 pin AVR ATtiny26 microcontroller. This microcontroller provides 16 controllable I/O pins. Each trace of the fabric PCB leads from one of the microcontroller's pins to one of the tabs visible on the top of the package. Each tab is labeled with the number of the pin it is connected to to assist users with electrical layouts and programming.
The entire package is approximately $51 \mathrm{~mm} \mathrm{x}$ $51 \mathrm{~mm}$. (The Lego piece, included in the pictures for scale, is approximately $16 \times 16 \times 10 \mathrm{~mm}$.) Each labeled tab is approximately $6 \mathrm{~mm}$ wide. Tabs are separated by $3 \mathrm{~mm}$ spaces to allow for easy hand or machine stitching.

To employ the microcontroller in an e-textile design, a user sews it into her textile. To make an electrical connection, she uses conductive thread to sew from one of its tabs to the component she wishes to control. (The silverized thread that is included in out kit will be discussed in section 2.5.) The resistance across a typical fabric tab to stitching joint is less than $1 \Omega$.
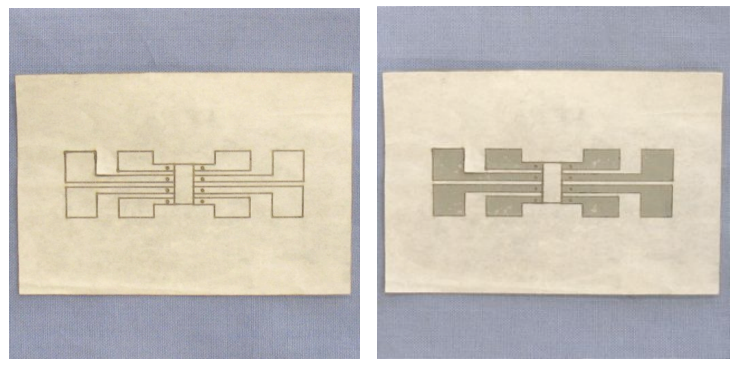

Figure 2. Constructing an iron-on circuit.

The fabric PCB is created with a technique developed at our lab that allows us to make "iron-on" circuits. To do this, we first attach a heat-activated adhesive to a conductive fabric, leaving the adhesive's paper backing attached to the conductive fabric. Then, we use a laser-cutter to score our circuit pattern into the conductive fabric, cutting the paper backing in the process (refer to the left hand image in Figure 2). We peel away the paper backing from the area underneath our circuit (refer to the right hand image in Figure 2). Using the sheet of conductive fabric to assist with placement, we iron our circuit onto a second piece of 

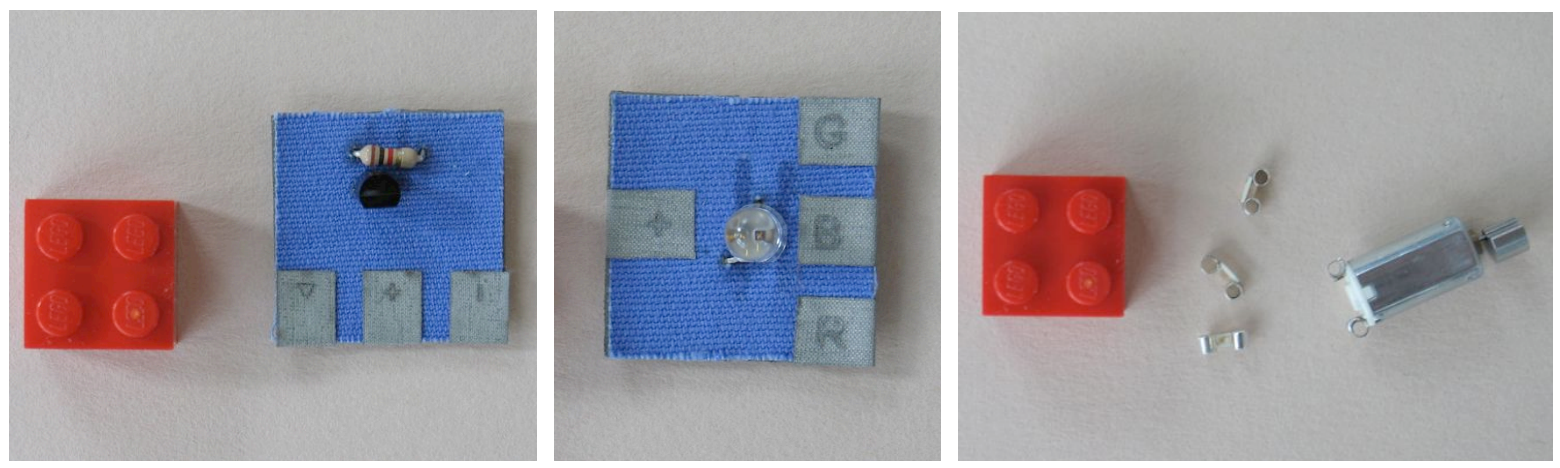

Figure 3. Left: a temperature sensor. Center: an RGB LED. Right: electronic sequins-LEDs and a vibrating motor.

(non-conducting) fabric. Only the parts of the conductive fabric where the paper has been removed adhere to this second piece of cloth. Finally, using the scored lines we etched on the laser-cutter, we peel away the conductive fabric that is not part of our circuit, leaving our fabric PCB attached to its cloth substrate.

The fabric we use for our iron-on circuits is an $\mathrm{Sn} / \mathrm{Cu}$ coated fabric manufactured by Less EMF [7]. We will discuss the wear characteristics of this cloth and our fabric PCBs in section 4

Once the circuit is attached to its backing cloth, it can be soldered like a traditional PCB. To create the stitch-able microcontroller package, we solder an IC socket to a fabric PCB and plug a microcontroller into the socket. The solder joints attaching the IC socket to the fabric PCB are protected with an encapsulation of clear epoxy resin as can be seen in Figure 1.

Programs for microcontrollers are typically written on a computer and then downloaded to a microcontroller via specialized hardware. This type of programming is generally perceived as a challenging activity requiring programming experience and familiarity with complicated hardware and software systems. We are currently investigating ways to help users overcome these challenges. At present, we are using AVR microcontrollers, the $\mathrm{C}$ programming language and the gcc compiler for our projects and workshops. We have developed $\mathrm{C}$ libraries to assist users with operations like controlling I/O pins, reading analogue data and communicating via IR. We use the STK500 board and UISP software to download programs to the microcontrollers.

We are concerned that even with the support of our libraries, the programming task could overwhelm novice users; our continuing work in this area may entail developing our own programming languages, development environments and programming hardware.

\subsection{Interaction: Sensors and Actuators}

To allow users to build interactive e-textiles, our kit contains an assortment of input and output devices: light, temperature and pressure sensors, light emitting diodes (LEDs) and vibrating motors. Figure 3 shows images of several of these devices.

We will begin our discussion of input/output (I/O) devices by examining sensors and in particular, the temperature sensor seen on the left in Figure 3. The temperature sensor is made, like the stitch-able microcontroller, with an iron-on circuit; it consists of a variable resistance temperature sensor and a resistor soldered to a fabric PCB. Electrical connections between the sensor and other components in an etextile design are made by stitching through the sensor's conductive fabric tabs. The tabs on the sensor indicate how it should be attached for proper reading; the + tab should be attached to Vcc, the - tab to ground, and the input tab to the place where sensor input will be read. The complete fabric sensor package is approximately $25 \times 25 \mathrm{~mm}$.

Our light sensor is built in an identical fashion, and other variable resistance sensors could be packaged in the same way. This packaging allows a user to use a sensor intuitively - he attaches it to his garment as it is labeled; he does not need to know how to wire a sensor to use one in his design.

The pressure sensor or touch switch, which can be seen in the lower right hand corner of the left hand image in Figure 1, was built using a technique first introduced by Post et al. [1]. Two pieces of conductive fabric (the two tabs protruding from the switch) are separated in the middle of the switch by a piece of felt with a hole in it. When the switch is pressed, the two pieces of conductive fabric contact one another through the hole. 

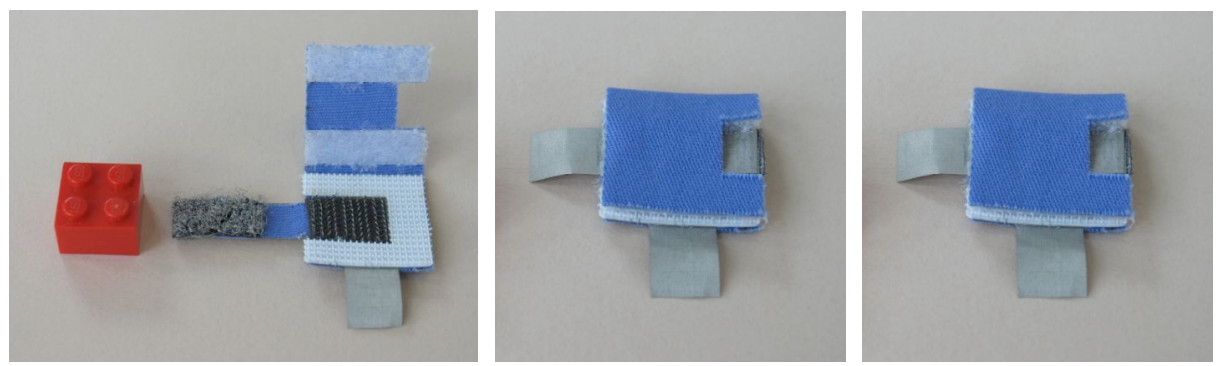

Figure 4. A soft on/off switch. Left: an open view. Center: the switch in its off position. Right: the switch in its on position.

Figure 3 also shows some of the output devices included in the kit: a red green blue (RGB) LED, single color LEDs and a vibrating motor. The RGB LED is packaged like the variable resistance sensors, with tabs indicating how it should be attached in a design. However, the other output devices are built in a different way.

Standard LEDs are easier to wire than sensors or RGB LEDs. No extra hardware is required to get them to work, and they have only two leads; a labeled fabric package is unnecessary and cumbersome. So, for simple components, we use our electronic sequin package: the LEDs and vibrating motor have been modified so that they can be sewn to fabric like beads or sequins. To build the "sequins" shown in Figure 3 we solder metal crimping beads to the leads of surface mount LEDs and motors. Similarly, we make through-hole components stitch-able by twisting their leads into a decorative spiral and leaving a hole in the spiral's center through which a needle can pass. The resistance across an average sequin to trace connection is less than $1 \Omega$.

\subsection{Communication: Infrared Transceiver}

To allow the e-textile designs that are built with the kit to communicate with other devices-PDAs and other textile designs, for example - the kit includes an IR transceiver module. Like the other fabric based sensor and actuator modules, it is a $25 \times 25 \mathrm{~mm}$ square with conductive fabric tabs that are labeled to indicate how the transceiver should be attached in a design.

\subsection{Power: Battery and On/Off Switch}

E-textile constructions require power. To fulfill this requirement, we use an off-the-self coin cell battery holder that can be sewn without any modifications (Keystone Electronics part \#1062). The holder comes in a surface mount package with leads that have holes in them, allowing the holder to be sewn. The holder has a footprint of approximately 25 x $22 \mathrm{~mm}$ and can hold two $20 \mathrm{~mm} 3 \mathrm{~V}$ batteries. Each battery can supply up to $220 \mathrm{mAh}$. Alternately, a single cell version of the holder can take one $3.6 \mathrm{~V}$ cell. We have found these power options to be sufficient for most of our applications. If devices require more amperage, holders can be tied together in parallel.

Our on/off switch, shown in Figure 4, is made entirely of fabric components. Conductive hook and loop surfaces are attached to conductive fabric tabs that are used to stitch the switch to constructions. When the hook and loop surfaces are joined, the switch is closed (on), when they are separated, the switch is open (off). When the switch is in the off position, a flap of non-conducting fabric separates the two contacts while allowing the switch to be folded into a compact configuration. When the switch is on, this flap is secured on top of the joined conducting surfaces. The resistance across a typical switch in its on position is also less than $1 \Omega$.

\subsection{Connection: Thread and Insulator}

In addition to the components shown in Figure 1, our kit also includes a spool of conductive thread, a needle and a tube of "puffy" fabric paint. We have been using a 234/34 x 4 silverized thread, manufactured by Shieldex [8], with a resistance of approximately $50 \Omega / \mathrm{m}$. The thread is too large to be used as top thread in a sewing machine, but works well when used as bobbin thread or as hand-sewing thread.

All of the electrical connections between components of our kit are made with stitching in conductive thread. This presents potential problems since exposed traces may come in contact with one another or other conducting surfaces as e-textile creations are worn. To address these issues, our kit includes a tube of thick ("puffy") fabric paint. When applied over conductive stitching, the paint functions as a durable and flexible insulator, preventing short circuits. 

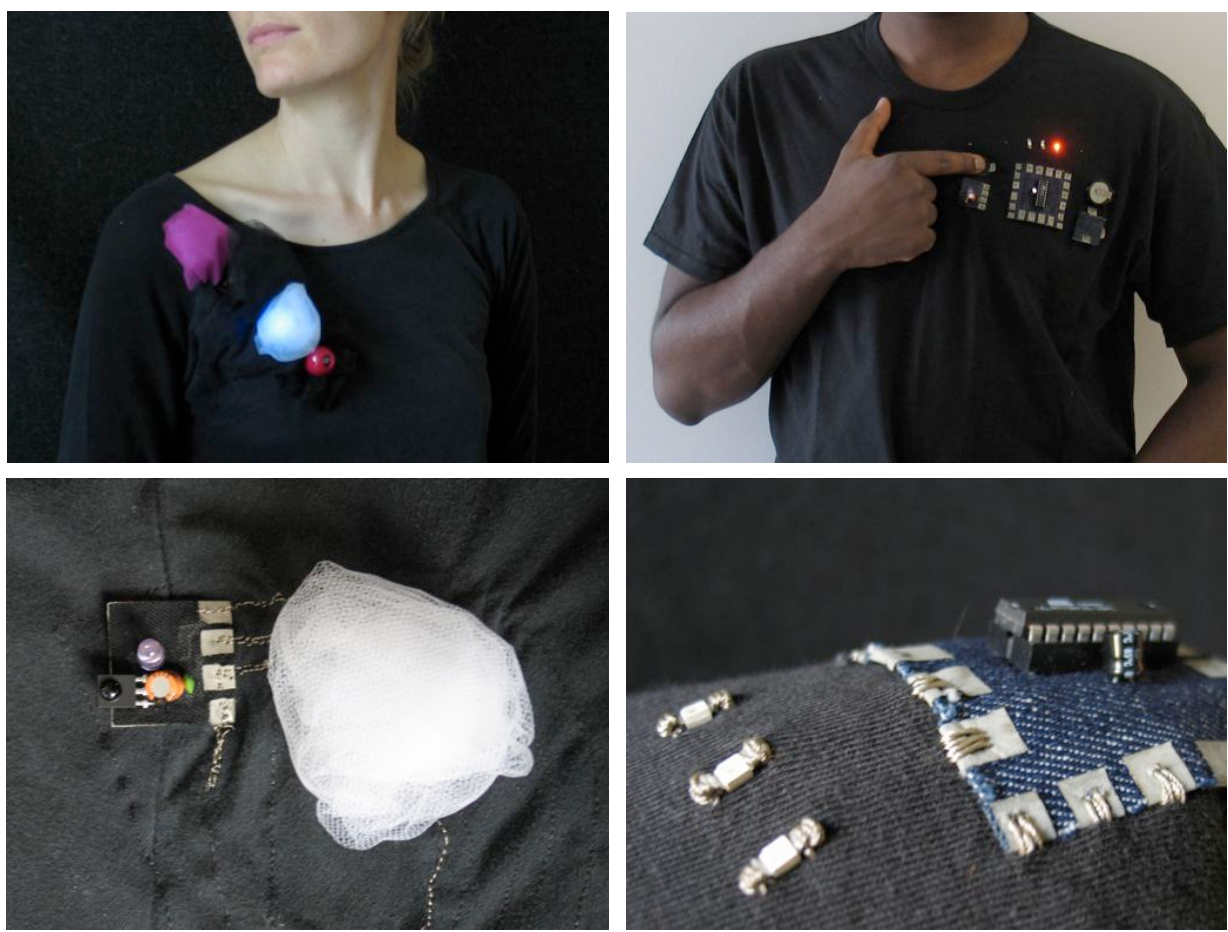

Figure 5. Top: Communicating shirts. Bottom: close-up views of the flower shirt before completion, and the stitching on the front of the man's shirt.

\section{Applications of the Kit}

To explore the range of constructions that can be built with the kit, we put together a few simple and playful wearable e-textiles, intended as examples of the types of items novices might build. This section will describe our creations: a set of communicating shirts, a temperature sensitive hat, and two wearable LED displays.

\subsection{Communicating Shirts}

To experiment with the kit's IR transceivers, we built two shirts that communicate with one another wirelessly via IR. These shirts can be seen in Figure 5. The first shirt has a decorative bundle of flowerlike shapes on its front. One of these houses an RGB LED, one a vibrating motor encased in a pink wooden bead, and one an IR transceiver. The second shirt has its hardware more exposed, as can be seen in the figure. This shirt contains an IR transceiver, a touch switch and three LED sequins.

Figure 5 also displays how the kit was employed to build the pieces. As has been described, components are connected to one another via stitching in conductive thread. We used a combination of hand and machine stitching to attach the components to the garments. Fabric PCB components are sewn via their conducting tabs, and electronic sequins are sewn like beads.

The functionality of the shirts is similar; each shirt is constantly "listening" for an IR signal. When the first shirt hears a signal, it replies to the message, turns on the vibrating motor to alert its wearer, and changes the color of the RGB LED to indicate the message it received. The second shirt sends out an IR signal whenever its switch is pressed. When the shirt receives a signal, it displays some information about the signal via its three LEDs.

One can imagine several scenarios for activities built around communicating shirts. For example, a shirt could be programmed to alert a wearer whenever a friend came within sight; a group of friends could program shirts to send identifying messages to each other. Each friend could be associated with a color, so that after a wearer was beeped, she could look down to see which friend was nearby.

\subsection{A Temperature-Sensing Hat}

Figure 6 shows a picture of a temperature-sensing hat that was constructed with the microcontroller, temperature sensor and RGB LED along with the on/off switch and battery. All of the electronic components except the RGB LED were mounted inside the hat so that they are hidden from view. 

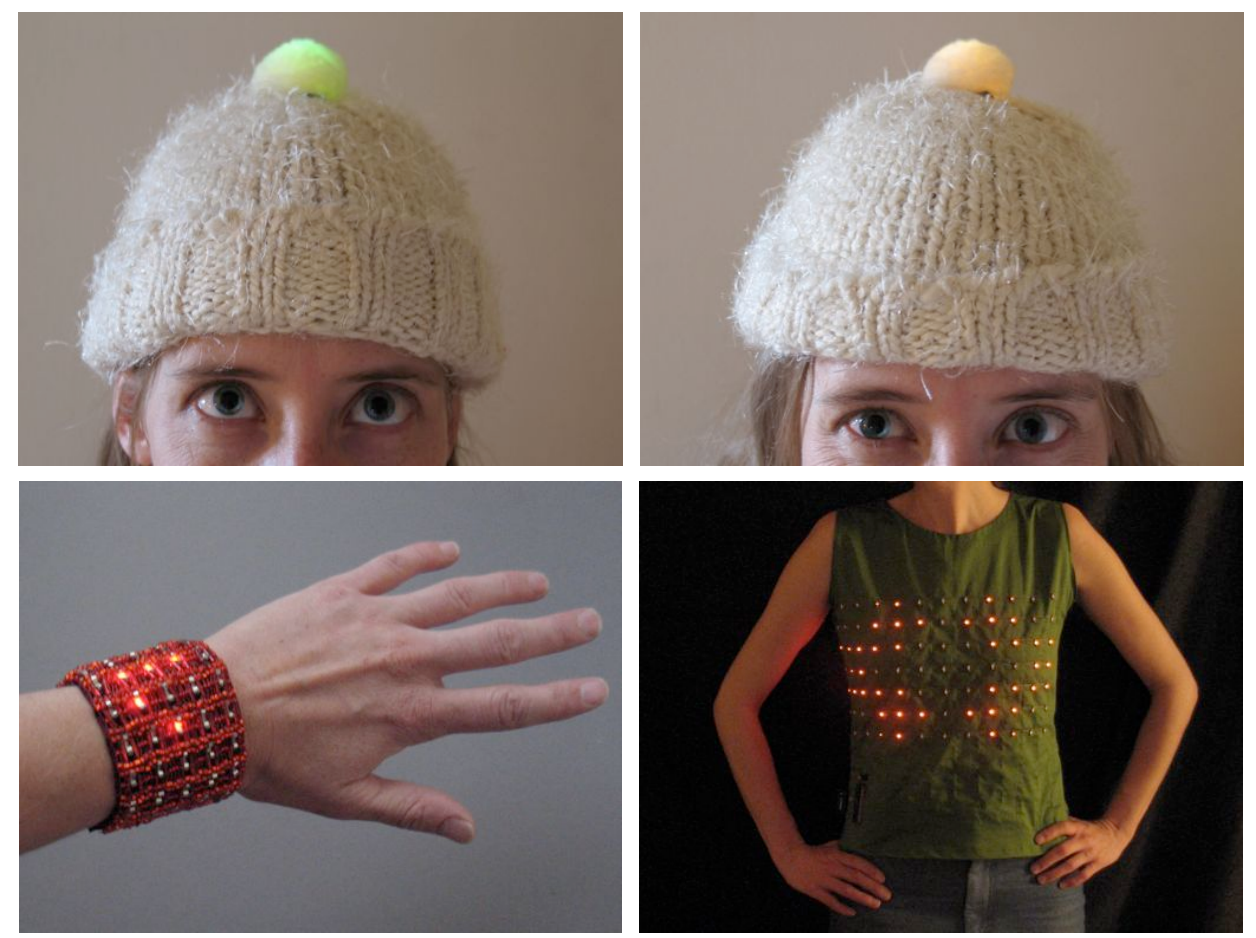

Figure 6. Top: A temperature-sensing hat. Bottom: Wearable LED displays.

The temperature sensor was attached so that it pokes through the knitting on the top of the hat to get a good reading. The RGB LED was stitched to the crown of the hat and then covered with a fluffy pompom that diffuses its light. As the temperature increases, the pompom gets redder; as the temperature decreases it gets bluer. At room temperature the pompom glows a yellowish green.

We wondered if the electronic components on the inside of the hat might feel awkward or catch in ones hair, but the cap is surprisingly comfortable. While not clearly visible in full sunlight, the LED can be seen outside on cloudy days or after dusk and in any indoor setting.

\subsection{Wearable LED Displays}

The wearable LED displays shown in Figure 6, a beaded LED sequin bracelet, and an LED sequin shirt, were built before we formalized our e-textile construction kit. They do not use the precise components that now make up our kit, but similar items could be built with the kit.

Each display contains a microcontroller and an array of LED sequins. The shirt also contains an IR receiver that facilitates communication between the shirt and a PDA. Each display can be programmed to display low-resolution animations like cellular automata or scrolling text.

We hope the examples we have presented illustrate how a wide range of projects can be built with the kit. The space of possible constructions ranges from ones that may take a few hours to build, (like the hat), to more sophisticated ones (like the displays) that may take a week or longer to complete.

\section{Durability}

The artifacts that are built with our kit must be truly wearable for our kit to be considered useful and useable. This section will describe our preliminary investigations into the durability of the components of the kit.

To test the robustness of the fabric PCB components, we washed a collection of components, including sensor and microcontroller packages, with a total of 104 encapsulated solder joints. We washed them 4 times in a standard commercial washing machine on the warm setting (water temperature approximately 20 degrees C/70 degrees F) with Arm and Hammer FabriCare detergent and dried them once in a standard commercial drier on the hot setting. 

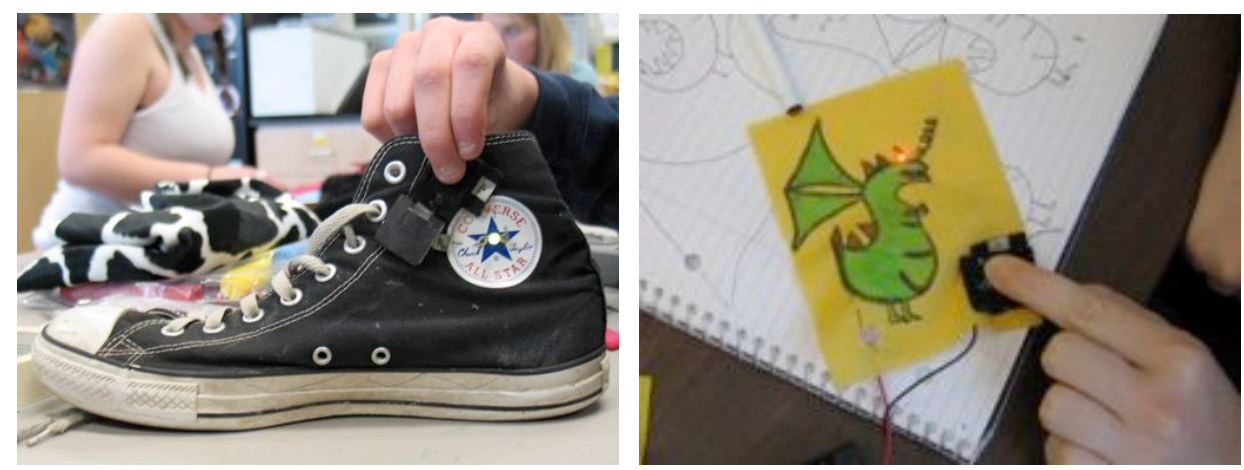

Figure 7. Cloth-based circuits built by participants in our user studies.

All of the solder joints survived this process. The resistance from solder joints to fabric did increase measurably, but in all cases remained below $1 \Omega$ after washing. It should be noted that Slade et al. [9] found the $\mathrm{Sn} / \mathrm{Cu}$ coated fabric we used to exhibit poor wear performance after 10 washing cycles and one drying cycle. More thorough testing is needed to determine how this fabric wears under various conditions.

A few fabric tabs did begin to peel up from their backing fabrics after our washing procedures. Additionally, several of the tabs cracked along the edge of the packages, where they were folded. While we anticipate that these issues may not pose large problems in garments where the tabs are sewn down, they could create difficulties in certain situations, and we plan to undertake investigations to find ways to prevent cracking and peeling.

To test our sequin packages, we washed five LED sequins stitched into cotton fabric with stainless steel thread. The sequins were washed five times, then dried, washed another five times and dried once more (for a total of 10 washings and 2 dryings). All of them withstood the washing and drying without breaking. We also tested an LED shirt similar to the one shown in Figure 6, but containing 84 LED sequins-we washed this shirt once and dried it once, and none of its sequins broke. Finally, we washed the shirt shown in Figure 6 by hand in cold water and allowed it to drip dry and it suffered no loss of functionality.

Our washing studies have not yet examined how the stitched interconnects between components wear; we have yet to was entire garments built with our kit. Further investigation is required to assess the reliability of stitched fabric tabs and sewn traces.

\section{Preliminary User Studies}

To determine the usability of our kit, we are conducting workshops in which novices work with the kit to build e-textile creations. We will begin this section by describing workshops we conducted with a simplified version of the kit and conclude with a brief discussion of our first experiences introducing the complete e-textile construction kit to students.

For the past year, we have held a series of electronic sewing workshops in which children and novice adults worked with a simplified version of the kit to build fabric-based electrical circuits and to augment existing e-textiles [10]. In these workshops, users were given needles, conductive thread, fabric switches, stitch-able batteries and LEDs. Participants worked with these components as well as traditional crafting materials to create their own electronic clothbased designs. Figure 7 shows products from some of these sessions.

We have held 9 workshops to date, with a total of approximately 80 participants. Each workshop lasted between $1 / 2$ and 2 hours. Participants ranged in age from 8-adult. Most of the participants (approximately $87 \%$ ) were able to complete working designs in the course of a workshop, though for many of them this was their first introduction to circuits.

In general, we have been encouraged by our experiences in the electronic sewing workshops. Users seem enthusiastic and curious about the activity and the medium. However, more detailed information is needed before we can draw any conclusions about the value of these experiences.

We are currently in the midst of a longer and more in-depth user study in which participants are using the complete e-textile construction kit. Through a local high school, we are teaching a course titled "Learn to Make Your Own Electronic Fashion". The class meets once a week for two hours for the duration of 8 weeks. We have 6 students, all high school freshman and sophomores, ranging in age from 14 to 16.

During the course of the class, the participants will use the kit described in this paper to build their own computationally controlled, interactive fashions. 
So far, the students have built simple circuits in fabric like the ones built in our electronic sewing workshops, (one student's construction is shown above in Figure 7), and have begun to work on microcontroller programs, using the $\mathrm{C}$ libraries and programming environment we described earlier. They have just begun to design their own complete e-textile pieces and will begin working with the rest of the e-textile components described in this paper soon.

\section{Social Implications and Areas for Future Study}

Computer science, electrical engineering, and related fields are overwhelmingly populated with males [11]. For example, the enrollment in the undergraduate computer science program at our university was $8 \%$ women for 2005 and the enrollment in the undergraduate electrical engineering program was even worse, at $7 \%$ women [12].

Though wary of stereotypes, we are excited by the possibility that the electronic textile medium may appeal more to women and girls than the media traditionally used to introduce embedded computing and electrical engineering and thus may provide a novel means for getting girls and women interested in these fields. Fuzzy, light-up clothes just look and feel very different from remote controlled cars or robots.

Very preliminary data seems to support these hypotheses. The high school where we are holding our electronic fashion class has a student population of 328 students. $52 \%$ of the students are male and $48 \%$ are female. Of the 6 students enrolled in our workshop, 5 are female and 1 is male, (perhaps because we included the word "fashion" in the title of our class?). Though it is impossible to draw conclusions from such a small sample size, we are intrigued by these numbers.

We hope to be able to undertake further investigation to determine if the experience of participating in a workshop like the one we are conducting could impact a person's attitudes towards technology related fields or their career choices.

In addition to gender concerns, we believe that there are a number of other interesting social issues to explore in using e-textiles as an educational medium. Kids and adults alike invest a tremendous amount of time and energy decorating themselves. If designers and educators can harness this energy, perhaps we can spark a popular e-textile hobbyist culture, increasing the technological literacy of students and inspiring the next generation of designers and engineers.

\section{Conclusion}

We think the possibilities for an e-textile construction kit are tremendously exciting. As the etextile field continues to flourish, we hope that our kit will help introduce people to the fascinating technological revolutions in textiles and computing that are taking place around them.

\section{References}

[1] R. Post et al. (2000) "E-Broidery: Design and fabrication of textile based computing", IBM Systems Journal, 39:34:840-860.

[2] The Georgia Tech wearable motherboard: The intelligent garment for the 21st century. Available:

http://www.smartshirt.gatech.edu

[3] P. Wilson (2005) "Textiles from novel means of innovation”, In McQuaid, M. (ed). Extreme Textiles: Designing for High Performance. Princeton Architectural Press. New York, NY, USA, 181-213.

[4] J. Berzowska (2005) "Electronic Textiles: Wearable Computers, Reactive Fashion, and Soft Computation", Textile, 3:1:2-19.

[5] M. Eisenberg et al. (2002). "Computationally-Enhanced Construction Kits for Children: Prototypes and Principles" In Proceedings of the Fifth International Conference of the Learning Sciences, 23-26.

[6] M. Resnick (2005) "Some Reflections on Designing Construction Kits for Kids", In Proceedings of Interactive Design and Children, 117-122.

[7] Less EMF. Available: http://www.lessemf.com

[8] Shieldex. Available: http://www.shieldextrading.net

[9] J. Slade et al. (2002) "Washing of Electrotextiles", In Proceedings of the Materials Research Society Symposium (MRS), 736:99-108.

[10] L. Buechley et al. (2006) Electronic/Computational Textiles and Children's Craft. In Proceedings of Interactive Design and Children, 49-56.

[11] J. Margolis and A. Fisher. (2001). Unlocking the Clubhouse: Women in Computing. MIT Press. Boston, MA, USA.

[12] University of Colorado Office of Planning, Budget and Analysis. Percent female enrolled by college, fall 2005. Available: http://www.colorado.edu/pba/div/FemalebyCol.htm 